\title{
Choix professionnels et représentations motivées du métier d'enseignant chez des étudiants en premier cycle
}

Jean-Claude Monnier

\section{OpenEdition}

Journals

Édition électronique

URL : http://journals.openedition.org/trema/2431

DOI : $10.4000 /$ trema.2431

ISSN : 2107-0997

Éditeur

Faculté d'Éducation de l'université de Montpellier

Édition imprimée

Date de publication : 1 mai 1992

Pagination : 47-59

ISSN : 1167-315X

Référence électronique

Jean-Claude Monnier, «Choix professionnels et représentations motivées du métier d'enseignant chez des étudiants en premier cycle », Tréma [En ligne], 1 | 1992, mis en ligne le 09 décembre 2013, consulté le 21 décembre 2020. URL : http://journals.openedition.org/trema/2431 ; DOI : https://doi.org/

10.4000/trema.2431

Ce document a été généré automatiquement le 21 décembre 2020.

Trema 


\title{
Choix professionnels et représentations motivées du métier d'enseignant chez des étudiants en premier cycle
}

\author{
Jean-Claude Monnier
}

1 Dans le cadre du Département de Formation des Maîtres de l'Université Paul Valéry de Montpellier, dirigé par Yveline Fumat, une enquête par questionnaire a été menée auprès d'un échantillon d'étudiants, au nombre de 256, se destinant aux métiers de l'éducation. Il s'agit d'étudiants de Lettres et Sciences Humaines qui s'inscrivent dans le Département des Sciences de l'Education et de la Formation de Montpellier III, pour y accomplir des modules de pré-professionnalisation.

\section{Sens et objectifs de ce travail d'enquête}

2 Il est apparu nécessaire, à la veille de modifications importantes de la formation des maîtres dans notre pays, de cerner les motivations des jeunes étudiants, en majorité issus du Lycée, en tout cas en cours de DEUG ou l'abordant, en fait pour la plupart en début de cursus universitaire, qui manifestent déjà des choix pré-professionnels dans le cadre de modules offerts à cet effet...

3 Le rapport Bancel souligne cette dimension pré-professionnelle: "[...] l'objectif est de permettre aux étudiants de s'orienter vers l'enseignement après un choix réfléchi et motivé... ».

4 Un questionnaire de réflexion et d'étude comportant, après identification de l'échantillon d'étudiants soumis à consultation, des questions à réponses pré-formées est proposé le 18 octobre 1990.

\section{Place et situation de cette investigation parmi d'autres enquêtes}

5 La nécessité d'un tel travail apparaît pleinement si nous nous référons au récent article de Marcel Postic («Motivations pour le choix de la profession d'enseignant », n91 avril- 
mai-juin 1990 de la Revue Française de Pédagogie) consacré à un public d'enseignants venant d'être reçus au concours de recrutement de professeurs d'enseignement général de lycée professionnel en 1987 lorsqu'il formule en introduction la remarque suivante : «L'enquête que nous présentons est faite auprès de personnes venant d'être reçues au concours de recrutement de professeurs d'enseignement général de lycée professionnel. Le choix de la profession d'enseignant est donc réalisé. Une autre investigation serait à mener auprès de jeunes étudiants qui choisissent de s'orienter vers l'enseignement afin de connaitre les raisons de leur projet, la représentation qu'ils ont de la profession et la perception des obstacles à la réalisation de leurs aspirations,... ».

\section{Radiographie de la population d'enquête}

\section{Qui sont-ils, ces étudiants, candidats aux métiers de l'éducation ?}

6 Nous pouvons avancer qu'il s'agit en très grande majorité de personnes de sexe féminin (pour $87 \%$ ), dont l'âge est compris entre 17 et 25 ans (pour $96 \%$ ), célibataires (à plus de $91 \%$ ), appartenant aux départements de l'Académie de Montpellier (à $78 \%$ ), vivant en chambre ou appartement personnel pour la moitié, chez les parents pour un sur trois, en Cité Universitaire pour près d'un un sur cinq ; $60 \%$ ont un Bac. littéraire, près de $20 \%$ un Bac. économie, mais pas d'autre diplôme, sinon pour une minorité le B.A.F.A.; ils proviennent d'une filière DEUG Psychologie et Lettres Modernes pour un sur quatre chacune, Langues étrangères pour près d'un sur cinq et Histoire-Géographie pour près d'un sur dix.

7 Enfin il s'agit d'étudiants dont la moitié ont les deux parents qui travaillent et l'autre moitié un seul parent ou aucun, dont les deux tiers sont issus des trois grandes catégories socio-professionnelles INSEE qui fournissent l'essentiel des masses étudiantes (cadres et professions intellectuelles supérieures, professions intermédiaires, artisans, commerçants et chefs d'entreprise) et pour un sur cinq provenant de la catégorie des employés ; peu d'étudiants sont d'origine rurale ou ouvrière.

\section{A quel métier aspirent-ils ?}

Le choix d'une UV préprofessionnelle ouvrant aux métiers de l'Education est plébiscité à $98 \%$; toutefois :

- un bon tiers manifeste de l'intérêt pour certaines formes du travail social ;

- un peu plus d'un sur huit seraient attirés par certains métiers de la santé.

En ce qui concerne les niveaux d'enseignement choisis, plus de $88 \%$ se fixeraient sur le professorat d'école, un peu plus d'un sur cinq se tourneraient vers le professorat de collège ou de lycée et très peu vers le secteur technique.

\section{L'image du métier d'enseignant compare à d'autres professions}

10 Il s'agit dans cette question de confronter l'image que l'on se fait du métier d'enseignant à 8 autres métiers plus ou moins rapprochés ou éloignés objectivement de lui sous quatre rubriques : intéressant, avantageux, utile à la société, tranquille. 


\section{Approche de la rubrique : « intéressant »}

Par rapport à enseignant le métier de :

\begin{tabular}{lcccccccr|}
\hline & Avocat & Artisan & Animateur & $\begin{array}{c}\text { Employé } \\
\text { de banque }\end{array}$ & Infirmier & $\begin{array}{c}\text { Interprète } \\
\text { d'armée }\end{array}$ & Capitaine Educateur \\
\hline $\begin{array}{l}\text { est... } \\
\text { plus } \\
\text { intéressant } \\
\text { pour... }\end{array}$ & $11,71 \%$ & 5,07 & 11,32 & 4,68 & 9,37 & 17,17 & 4,29 & 18,75 \\
\hline $\begin{array}{l}\text { moins } \\
\text { intéressant } \\
\text { pour... }\end{array}$ & $36,71 \%$ & 55,85 & 15,62 & 87,10 & 41,79 & 43,75 & 84,76 & 3,90 \\
$\begin{array}{l}\text { autant } \\
\text { intéressant } \\
\text { pour... }\end{array}$ & $47,26 \%$ & 34,76 & 70,31 & 5,46 & 46,09 & 37,10 & 7,03 & 75,78 \\
\hline $\begin{array}{l}\text { Non réponses } \\
\text { 3, }\end{array}$ & $3,90 \%$ & 3,51 & 1,95 & 2,34 & 2,34 & 3,90 & 3,51 & 1,17 \\
\hline
\end{tabular}

\section{Commentaires}

121 - Il n'y a qu'un dixième à un cinquième à peine d'étudiants qui jugent les métiers d'éducateur, d'interprète, d'avocat et d'animateur plus intéressants que celui d'enseignant; par contre très peu d'attrait se manifeste pour capitaine, employé de banque, artisan ; le métier d'infirmier ne recueille même pas un dixième de l'échantillon.

13 C'est donc très majoritairement que le métier d'enseignant est posé comme plus intéressant, tant des métiers qui lui sont proches par la similarité des intérêts éducatifs qu'ils peuvent partager, que des métiers qui s'en éloignent de par les besoins sociaux différents qu'ils remplissent...

2 - Ce point de vue est largement confirmé par le rejet des métiers jugés moins intéressants : rejet manifeste de l'employé, du capitaine, de l'artisan ; rejet à un degré moindre mais non négligeable de l'interprète, de l'infirmier, de l'avocat; enfin peu de rejet pour les métiers voisins de celui d'enseignant, à savoir les métiers d'animateur et d'éducateur qui entrent davantage en concurrence parmi les possibles offerts au projet professionnel de l'étudiant.

\section{Approche de la rubrique " avantageux »}


Par rapport à enseignant le métier de :

\begin{tabular}{|c|c|c|c|c|c|c|c|c|}
\hline & Avocat & Artisan & Animateur & $\begin{array}{l}\text { Employé } \\
\text { de banque }\end{array}$ & Infirmier & $\begin{array}{l}\text { Interprète } \\
\text { d'armée }\end{array}$ & Capitaine & Educateur \\
\hline $\begin{array}{l}\text { est... } \\
\text { plus } \\
\text { avantageux } \\
\text { pour... }\end{array}$ & $61,79 \%$ & 12,89 & 3,90 & 41,40 & 10,54 & 50,39 & 46,09 & 8,59 \\
\hline $\begin{array}{l}\text { moins } \\
\text { avantageux } \\
\text { pour... }\end{array}$ & $19,53 \%$ & 65,62 & 45,70 & 33,20 & 45,31 & 19,53 & 38,67 & 24,60 \\
\hline $\begin{array}{l}\text { autant } \\
\text { avantageux } \\
\text { pour... }\end{array}$ & $14,06 \%$ & 16,01 & 46,48 & 20,31 & 38,67 & 23,83 & 6,25 & 61,32 \\
\hline Non réponses & $4,29 \%$ & 5,07 & 3,90 & 5,07 & 5,46 & 5,85 & 8,97 & 5,46 \\
\hline
\end{tabular}

\section{Commentaires}

151 - Si le métier d'enseignant restait, par rapport à n'importe quel autre métier ici mentionné, le plus intéressant, le métier d'avocat est perçu comme nettement plus avantageux avec près de $62 \%$ des réponses de l'échantillon; puis à un degré moindre, pour la moitié de l'échantillon, le métier d'interprète est aussi perçu comme plus avantageux.

On remarquera que capitaine d'armée et employé de banque réalisent des pourcentages relativement appréciables avec respectivement plus de $45 \%$ et plus de $41 \%$ des réponses. Seuls les métiers d'éducateur et d'animateur tombent en dessous de $10 \%$ des réponses.

2 - Seul le métier d'artisan est perçu comme véritablement moins avantageux à une très forte majorité des deux tiers $(65,62 \%)$ : cette image négative peut nous laisser penser qu'une vision strictement quantitative des métiers ne saurait suffire, confirmée en cela par une appréciation négative de capitaine d'armée pour $39 \%$ de l'échantillon.

Par contre les moindres avantages accordés aux métiers d'animateur (plus de $45 \%$ ) et d'infirmier (plus de $45 \%$ également) conjuguent-ils à la fois des éléments quantitatifs et qualitatifs pour constituer leur image négative? Image négative très relative car elle est contrebalancée nettement pour celle d'animateur d'une équivalence d'avantages d'avec le métier d'enseignant pour plus de $45 \%$ de l'échantillon. C'est aussi le cas mais à un degré moins ambigu pour l'image négative d'infirmier qui le reste avec seulement $38 \%$ de jugements équivalents.

\section{Approche de la rubrique " utile à la société »}


Par rapport à enseignant le métier de :

\begin{tabular}{|c|c|c|c|c|c|c|c|c|}
\hline & Avocat & Artisan & Animateur & $\begin{array}{l}\text { Employé } \\
\text { de banque }\end{array}$ & Infirmier & $\begin{array}{l}\text { Interprète } \\
\text { d'armée }\end{array}$ & Capitaine & Educateur \\
\hline $\begin{array}{l}\text { est... } \\
\text { plus } \\
\text { utile à la } \\
\text { société } \\
\text { pour... }\end{array}$ & $8,59 \%$ & 5,46 & 6,25 & 8,20 & 29,68 & 7,42 & 8,20 & 13,27 \\
\hline $\begin{array}{l}\text { moins } \\
\text { utile à la } \\
\text { société } \\
\text { pour... }\end{array}$ & $10,54 \%$ & 38,67 & 32,42 & 58,20 & 1,17 & 47,26 & 65,23 & 4,29 \\
\hline $\begin{array}{l}\text { autant } \\
\text { utile à la } \\
\text { société } \\
\text { pour... }\end{array}$ & $76,56 \%$ & 50,39 & 55,25 & 30,46 & 66,01 & 38,30 & 21,86 & 78,51 \\
\hline Non réponses & $4,29 \%$ & 5,07 & 4,68 & 3,12 & 2,73 & 7,03 & 4,68 & 3,90 \\
\hline
\end{tabular}

\section{Commentaires}

1 - Si l'on excepte infirmier, jugé plus utile que le métier d'enseignant par près de $30 \%$ de l'échantillon, les autres métiers ne sont jugés tels généralement par moins de $10 \%$ de l'échantillon hormis éducateur qui obtient un peu plus de $13 \%$ : l'idée d'utilité sociale au sens de service public ou de servir autrui reste une idée forte pour le métier d'enseignant. 2 - Sont jugés plutôt massivement moins utiles : le capitaine d'armée pour plus de 65 \% et l'employé de banque pour plus de $58 \%$. Puis de façon moins majoritaire l'interprète avec plus de $47 \%$, suivi de l'artisan avec plus de $38 \%$ et de l'animateur pour près d'un tiers de l'échantillon (32,42\%).

On notera qu'infirmier jouit a contrario d'une forte image d'utilité sociale en concurrence avec celle d'enseignant : à peine un peu plus d' $1 \%$ juge moins utile la fonction d'infirmier. 3 - Parmi les métiers jugés autant utiles que celui d'enseignant on remarquera :

- les scores se situant au-delà des trois quarts de l'échantillon où l'on trouve en tête éducateur avec plus de $78 \%$, suivi d'avocat avec plus de $76 \%$;

- l'important score d'infirmier avec plus des deux tiers de l'échantillon et ce, en cohérence avec cette image de forte utilité que nous avons déjà souligné en 3-1 et 3-2 où se marque bien cette concurrence avec le métier d'enseignant sur ce registre du service public et du service à autrui ;

- le score non négligeable d'animateur pour plus de $55 \%$ de l'échantillon mais qu'il faut mettre en balance avec le tiers qui l'a jugé moins utile précédemment ;

- celui d'artisan avec un peu plus de $50 \%$ est davantage encore mis en balance avec les plus de $38 \%$ qui le jugeaient moins utile auparavant ;

- enfin interprète et plus nettement encore employé de banque ont des balances négatives assez marquées... quant à leur utilité sociale comparée avec celle d'enseignant.

\section{Approche de la rubrique « tranquille»}


Par rapport à enseignant le métier de :

\begin{tabular}{|lcccccccr|}
\hline & Avocat & Artisan & Animateur & $\begin{array}{c}\text { Employé } \\
\text { de banque }\end{array}$ & Infirmier & $\begin{array}{l}\text { Interprète Capitaine Educateur } \\
\text { d'armée }\end{array}$ & \\
\hline $\begin{array}{l}\text { est... } \\
\text { plus } \\
\text { tranquille } \\
\text { pour... }\end{array}$ & $12,5 \%$ & 40,6 & 16,4 & 70 & 3,5 & 43 & 42,6 & 2,7 \\
\hline $\begin{array}{l}\text { moins } \\
\text { tranquille } \\
\text { pour... }\end{array}$ & $54,7 \%$ & 39 & 24,2 & 12,9 & 75,4 & 28,5 & 41 & 44,9 \\
$\begin{array}{l}\text { autant } \\
\text { tranquille } \\
\text { pour... }\end{array}$ & $26,9 \%$ & 15,2 & 48,4 & 13,7 & 16,4 & 24,6 & 10,5 & 47,6 \\
\hline $\begin{array}{l}\text { Non réponses } \\
5,8 \%\end{array}$ & 5 & 7 & 3,1 & 4,7 & 7,4 & 6 & 5 \\
\hline
\end{tabular}

\section{Commentaires} plus tranquille de tous reproduisant ou reprenant à leur compte l'image stéréotypée que l'ensemble de la société véhicule, celle d'une profession sans grande prise de risque sinon routinière et répétitive dans ses actes,

24 A un degré moindre mais de façon non négligeable trois autres métiers sont jugés par plus de $40 \%$ des étudiants comme plus tranquilles que celui d'enseignant, respectivement interprète pour $43 \%$, capitaine d'armée pour $42,6 \%$, artisan pour $40,6 \%$ : on peut penser que cette représentation similaire de trois métiers aussi différents entre eux repose soit sur une certaine méconnaissance des réalités de ces métiers comme interprète ou artisan soit sur des motifs eux-mêmes différents; l'image tranquille du capitaine d'armée peut trouver sa raison dans l'appartenance à la catégorie des fonctionnaires et à un équilibre conjoncturel de paix.

On remarquera, par contre, que les images des métiers d'éducateur avec $2,7 \%$ et d'infirmier avec 3,5\% tels qu'ils sont perçus nous les rendent comme fort peu tranquilles.

2 - Ce point de vue est largement confirmé pour infirmier puisque les trois quarts des étudiants $(75,4 \%)$ se le représentent comme le moins tranquille des huit métiers proposés dans leur rapport avec celui d'enseignant.

Suivra en second lieu celui d'avocat pour lequel 54,7 \% des étudiants le jugent aussi moins tranquille que le métier d'enseignant: on peut penser que ces deux métiers, infirmier et avocat, sont jugés comme comportant des prises de risque engageant jusqu'à la vie d'un être humain.

Cette même représentation du degré dans la prise de risque peut faire comprendre pourquoi $44,9 \%$ des étudiants jugent « éducateur » comme moins tranquille et davantage encore, à savoir $47,6 \%$, se le représentent comme autant tranquille que le métier d'enseignant. Ici, la communauté de vue très forte de ces deux métiers de l'éducation l'emporte pour les situer en second sur une échelle hypothétique de prise de risque. 
On remarquera, par ailleurs, que le troisième métier de l'éducation, proche de ces deux premiers, celui d'animateur est crédité par un pourcentage équivalent d'étudiants, soit $48,4 \%$ d'autant de tranquillité que celui d'enseignant.

\section{Besoin et motivations dans le choix du métier d'enseignant}

La recherche du besoin préférentiel dans le métier relève du domaine des motivations. Celles-ci peuvent se définir comme l'ensemble des phénomènes dont va dépendre la stimulation à agir pour atteindre un objectif donné.

D'où nous conviendrons que chacun des participants au questionnaire est caractérisé par la dominance d'un ou de deux de ces cinq besoins proposés, que ceux-ci existent certainement chez chacun mais en des proportions différentes; ce qui autorise un classement et l'élaboration d'un tableau qui nous donne les dominantes du groupe considéré : c'est-à-dire besoins ayant les valeurs 5 et 4 ou besoins dominants, une valeur moyenne (côté 3 ) et des besoins moins marquants (côtés 2 et 1 ).

Quels sont pour notre échantillon les besoins dominants et les besoins non dominants qui motiveraient le choix du métier? Sont-ils différents ou non de ceux exprimés par les enseignants actuellement en poste? Varient-ils fortement ou non de la représentation que s'en fait la population française?

Voici les résultats de notre enquête.

\begin{tabular}{|lccccc|}
\hline & Sécurité & Affection & Prestige & Considération & Epanouissement \\
\hline $\begin{array}{l}\text { Valeur dominante } \\
\text { (côté 5 et 4) }\end{array}$ & $39,61 \%$ & 30,69 & 8,65 & 29,12 & 87,33 \\
$\begin{array}{l}\text { Valeur moyenne } \\
\text { (côtée 3) }\end{array}$ & $35 \%$ & 29,13 & 4,33 & 26,37 & 4,72 \\
\hline $\begin{array}{l}\text { Valeur de moindre } \\
\text { importance } \\
\text { (côtée 2 et 1) }\end{array}$ & $23,45 \%$ & 38,18 & 85,03 & 42,51 & 7,08 \\
\hline
\end{tabular}

\section{Commentaires}

1 - Le besoin d'épanouissement dans le métier ou besoin d'accomplissement de soi, selon la théorie des besoins hiérarchisés de Maslow (1943) - besoin situé au niveau le plus élevé de la hiérarchie selon cet auteur - constitue bien le besoin essentiel visé et recherché par les étudiants se destinant aux métiers de l'éducation. Avec plus de $87 \%$ (dont plus de $78 \%$ en valeur 5), c'est un besoin largement plébiscité puisqu'il recueille plus du double des suffrages, comparé au besoin de sécurité qui vient en seconde position avec seulement près de $40 \%$ des choix en valeur dominante (dont plus de $31 \%$ qu'en valeur 4 );

352 - Le besoin de sécurité également choisi comme valeur dominante par le groupe soumis à enquête reste une valeur dominante choisie moins appréciée mais marquante cependant :

- en raison d'abord des $35 \%$ des suffrages en valeur moyenne qui lui sont accordés, puis de la différence significative, de plus de 16 points, entre les cotations 5 et 4 et les cotations 2 et 1 ; 
- par la signification qu'il peut revêtir. Est-il la marque d'une inquiétude profonde face à un avenir professionnel incertain que l'on pense conjurer par un choix socio-professionnel posé comme relativement stable dans nos pays où le besoin de matière grise va croissant ? Ou bien le signe de la recherche d'une relative quiétude grâce à l'existence d'un statut jusqu'alors toujours encore socialement protégé tout en étant susceptible de conjuguer emploi administratif avec une certaine liberté d'initiative et de rythme de vie?

3 - Parmi les besoins moins déterminants, celui de prestige est le plus largement rejeté avec plus de $85 \%$ des suffrages (dont plus de $77 \%$ en valeur 1). Il en est de même mais à un degré bien moindre du besoin de considération qui est rejeté par plus de $42 \%$ (dont plus de $39 \%$ en valeur 2), rejet qui est tout de même moitié moins fort que celui du besoin de prestige. Si le rejet du prestige est sans ambiguïté, on ne peut en dire autant de celui du besoin de considération qui, avec près de $30 \%$ en valeurs 5 et 4 , offre un score de choix positifs plus important que la valeur moyenne obtenue $(26 \%)$. On remarquera que la somme des valeurs 5,4 et 3 , en renversant la tendance, accuse encore plus cette ambivalence. Comment expliquer le très faible impact du besoin de prestige et le caractère ambigu du choix du besoin de considération ? :

- d'abord constatons que le jeune étudiant n'opte pas pour les métiers d'éducation parce que ceux-ci offriraient prestige et valorisation sociales. Ce résultat, n'est-il pas paradoxal alors qu'a souvent été relevée et soulignée depuis au moins 2 ou 3 décennies l'image socialement dévalorisée des enseignants dans tout l'hexagone, l'évolution s'opérant à l'avantage du cadre du secteur privé ? Cette perte de prestige est par contre nettement ressentie par le corps enseignant lui-même (déjà une enquête publiée en 1973 par la Documentation Française auprès d'enseignants du second degré révélait que $89 \%$ des sujets alors interrogés reconnaissait cette perte de prestige comme signe d'un déclassement social). On retrouverait dans la revendication des maîtres du premier degré, le souhait d'une « égale dignité » dans la promotion vers le corps unique des enseignants, une tendance tout à fait similaire, signe d'un déclassement social nettement ressenti...

- Ensuite il faut marquer une nette différence entre besoin de prestige et besoin de considération, bien traduite ici par la différence nette de pourcentages : l'ampleur des réponses négatives à l'égard du besoin de prestige prouve que les jeunes étudiants ne sont pas mus par le désir d'acquérir un statut social prestigieux toujours signe de puissance ou de reconnaissance sociale jugée superficielle ou de simple façade... Il est heureux que le personnage ne puisse l'emporter sur la personnalité vraie...

Par contre les choix plus ambigus touchant au besoin de considération sociale, même s'il reste également refusé en majorité relative ne traduit-il pas la situation temporaire du jeune étudiant plutôt qu'une évolution à contre-courant. A savoir :

- d'abord le besoin de considération c'est-à-dire d'estime, de respect et de reconnaissance de la part d'autrui peut exprimer simplement le souhait d'être reconnu pour ce que l'on est et ce que l'on fait (ou fera) dans son propre métier : si le prestige est en soi récusé, être apprécié pour son activité professionnelle - ce qui ne peut être qu'anticipé par les jeunes qui s'y destinent - reste au demeurant parfaitement légitime... Que l'on songe à ce besoin de considération professionnelle qui seule peut fonder un rapport de confiance vis-à-vis des parents d'élèves par exemple !

- ensuite ce besoin mitigé de considération sociale est peut-être construit davantage sur la base d'un rôle social à jouer, y compris à promouvoir, si l'on est conscient ou informé des situations socialement et économiquement objectives, que sur celle d'un statut social tout tracé d'avance ; peut-être que même à statut socio-économique peu considéré c'est la 
fonction sociale jouée qui primerait, y compris en opérant selon un mécanisme compensatoire : peu sensibles encore aux enjeux économiques ou de puissance à la façon des cadres en prise directe sur les réalités - et non encore en exercice professionnel réel - les jeunes étudiants seraient, semble-t-il, plus sensibles à plus de sécurité - ce que nous avons vu précédemment - et se révèleraient plus désintéressés en faisant de la valeur d'épanouissement leur réalisation ultime... jugé, avec une plus faible majorité encore que ne l'était le besoin de considération, comme moins déterminant dans le choix professionnel avec seulement $38 \%$ des choix (dont plus de $28 \%$ en valeur 2 ). voir, positive avec plus de $30 \%$ et moyenne avec près de $30 \%$ ). Tentons une explicitation probable des 3 modes de réponses :

- la valeur centrale moyenne constitue généralement une valeur refuge traduisant soit « je ne peux » soit « je ne veux » pas répondre face à cette ambivalence marquée ;

- la valeur de refus peut-être analysée : soit comme une attitude de lucidité à l'égard des pièges toujours possibles de l'affectivité, soit comme une attitude réaliste et distanciée considérant que le choix professionnel ne saurait reposer sur de telles orientations par nature plus incontrôlées que réfléchies ; à moins que ne l'emporte l'attitude très réservée à l'égard d'autrui duquel on ne saurait attendre de retour d'affect soit encore que la relation d'enseignement se trouve déjà posée comme distanciée par nécessité (médiatisée qu'elle est par son objet « le contenu-matière » à transmettre), soit enfin que la relation d'enseignement tend déjà à se protéger des possibles envahissements des phénomènes de groupe...;

- quant à la valeur de choix positif elle traduirait plutôt la nécessaire prise en compte qu'un métier où règne en permanence le relationnel - disons même malgré soi - les motivations altruistes souvent avancées comme motifs (aider les enfants et les jeunes, favoriser leur épanouissement ou leur développement ou leur expression, s'estimer soi-même en estimant les autres, etc.) s'alimentent forcément d'une réciprocité dynamique, sans laquelle aucune évolution majorante ne semble possible pour les deux sujets en présence. C'est aussi reconnaître, à leur place, la part non négligeable, à côté des motivations de nature proprement intellectuelle, des identifications qui sont en jeu de part et d'autre, dès lors que l'on prend la mesure que toute transaction d'enseignement est toujours une rencontre de sujets qui s'éprouvent et « s'affectent »...

5 - Une comparaison concernant ces mêmes besoins, tels qu'ils sont perçus, à propos des métiers de l'enseignement, par les enseignants eux-mêmes et la population française peut offrir un éclairage intéressant.

Il s'agit des enquêtes menées par la D.I.C. (Direction de l'Information et de la Communication) du Ministère de l'Education Nationale en 88-89 dont on trouvera les résultats qui nous intéressent dans le tableau ci-après : 
Comparaison des perceptions du métier d'enseignant telles qu'elles résultent de l'analyse de l'appréciation de la population enseignante, de la population française et de la population étudiante de notre enquête.

\begin{tabular}{|lcccccc|}
\hline & $\begin{array}{c}1 \\
\text { Population } \\
\text { française }\end{array}$ & $\begin{array}{c}2 \\
\text { Enseignants }\end{array}$ & $\begin{array}{c}\text { Ecarts entre } \\
1 \text { et 2 }\end{array}$ & $\begin{array}{c}\text { Etudiants } \\
\text { (\% arrondis) }\end{array}$ & $\begin{array}{c}\text { Ecarts entre } \\
1 \text { et 3 }\end{array}$ & $\begin{array}{c}\text { Ecarts entre } \\
\text { 2 et 3 }\end{array}$ \\
\hline $\begin{array}{l}\text { Besoin de } \\
\text { sécurité } \\
\begin{array}{l}\text { Besoin } \\
\text { d'affection }\end{array}\end{array}$ & $55 \%$ & $38 \%$ & -17 & $40 \%$ & -15 & +2 \\
$\begin{array}{l}\text { Besoin de } \\
\text { prestige }\end{array}$ & $47 \%$ & $48 \%$ & +1 & $31 \%$ & -16 & -17 \\
\hline $\begin{array}{l}\text { Besoin de } \\
\text { considération }\end{array}$ & $17 \%$ & $20 \%$ & +3 & $30 \%$ & +13 & +10 \\
\hline $\begin{array}{l}\text { Besoin } \\
\text { d'épanouissement }\end{array}$ & $30 \%$ & $49 \%$ & +19 & $88 \%$ & +58 & +39 \\
\hline
\end{tabular}

\section{Commentaires}

Si les étudiants se destinant aux carrières de l'éducation et les enseignants en poste sont en phase en ce qui concerne la recherche du besoin de sécurité et ces deux populations en décalage avec la perception que s'en fait la population française, plus surprenant apparaît le déficit dans le registre du besoin d'affection alors qu'enseignants et population française sont là particulièrement en phase.

Par contre l'écart devient massif entre population étudiante et population française et enseignante concernant le besoin de prestige, écart qui va croissant au regard de la population française qui n'était déjà plus en accord avec les représentations de la population enseignante...

4 Inversement le besoin d'épanouissement qui distinguait déjà nettement les enseignants de l'ensemble de la population française creuse davantage plus nettement encore la distance séparant les étudiants tant avec la population enseignante qu'avec la population française.

Enfin le besoin de considération semble davantage une préoccupation de la population étudiante que des enseignants eux-mêmes ou de l'ensemble de la population française...

Hormis le besoin de sécurité, on voit mieux ce qui distingue population enseignante, en poste, et population étudiante se destinant aux carrières de l'éducation :

- la première accorde d'abord autant d'importance au besoin d'épanouissement qu'au besoin d'affection comme si l'un et l'autre étaient en relation étroite et dialectique ; puis - mais avec une importance moindre mais non négligeable - donne autant d'importance au besoin de prestige qu'au besoin de sécurité ; enfin tend à négliger le besoin de considération : ainsi se marque une certaine recherche d'équilibre entre différentes valeurs socioprofessionnelles et personnelles pour réaliser le profil de l'enseignant d'aujourd'hui ;

- la seconde tend au contraire à récuser à l'extrême le besoin de prestige puis à l'autre extrême à plébisciter le besoin d'épanouissement ; ensuite à accorder de l'importance au 
besoin de sécurité ; enfin à équilibrer le besoin d'affection et besoin de considération en y donnant une importance relative non négligeable...

Ce décalage population enseignante/population étudiante qui touche 4 besoins sur 5 que signifie-t-il ?:

- marque-t-il simplement la différence de ceux qui sont déjà confrontés aux réalités du métier et de ceux qui ne font qu'y aspirer avec ce qui ne seraient que les élans de la jeunesse ?

- ou bien peut-on y percevoir le signe d'une nouvelle génération montante à la recherche d'un nouvel équilibre des valeurs socio-professionnelles et personnelles?

En conclusion les résultats révèlent :

- un attrait pour l'enfance qui fixe majoritairement les choix des étudiants vers le professorat d'école, à un moment où l'inversion de la courbe des besoins exige, au contraire, d'ici l'an 2000, davantage de professeurs du secondaire ;

- sans conteste, une adhésion au métier d'enseignant qui apparaît à leurs yeux comme le plus intéressant, même s'il n'est pas le plus avantageux, et comme porteur d'une grande utilité sociale mais non perçu comme tranquille; c'est dire qu'il génère l'image positive mais lucide d'un métier ouvert aux autres et comportant une certaine prise de risque et de responsabilité qu'on entend bien assumer ;

- enfin un ensemble motivationnel pour ce métier, à caractère plus intrinsèque qu'extrinsèque, en décalage avec la population enseignante en poste aujourd'hui, qui traduirait, semble-t-il, chez ces jeunes étudiants, de nouveaux motifs d'investir ce métier et de s'y accomplir à leur façon.

\section{BIBLIOGRAPHIE}

BANCELDaniel. Rapport au M.E.N. « Créer une nouvelle dynamique de la formation des maîtres », texte multigraphié, 10 octobre 1989.

BENEDETTO Pierre. «Intérêts, maturité vocationnelle et choix des études. » L'Orientation scolaire et professionnelle, vol. 16, n³, 1987, pp . 193-206.

MASLOW A.H. Motivation and personality. New York, Harper, 1970.

MAC CLELLAND D.C. Human Motivation. Glenview, ill. Scott, Foresman and Co, 1985.

MUCCHIELLIRoger. Le questionnaire dans l'enquête psycho-sociale. Paris, ESF, 1970.

Ministère de l'Education Nationale, Direction de l'Information et de la Communication. Enquêtes.

- Brochure « Regard des jeunes sur le système éducatif ».

- 4 fascicules : «L'opinion des français et des parents d'élèves sur l'école »; « Les enseignants : perception de l'école et de leur métier »; «Les jeunes et leur établissement scolaire »; « Les chefs d'entreprise et le système éducatif ». 36-15 Educatel. Code Regard.

Postic Marcel et coll. Motivations pour le choix de la profession d'enseignant. Revue Française de Pédagogie, n 91, 1990, pp. 25-36, Paris. 


\section{RÉSUMÉS}

L'enquête menée auprès d'étudiants de Premier cycle universitaire de Lettres et Sciences humaines, à la rentrée 1990, s'efforce de cerner, chez ceux qui se destineraient aux métiers de l'Education, à la fois leur préférence professionnelle, l'image qu'ils se font du métier d'enseignant parmi d'autres métiers possibles et les motivations qui les animent dans ces choix. Les choix se manifestent nettement en faveur de l'enfance et du professorat d'école, à la faveur d'une vision positive mais lucide du métier.

At the beginning of the 1990 university year a survey of undergraduate students in Letters and Humanities aims at revealing their professional preferences as well as the image they have of a teaching profession as opposed to other possible professions and their motivation concerning their choice. These students were all destined to an educational profession. The choices reveal a preference for childhood and the profession of a school teacher in favour of a positive but realistic vision of the profession.

\section{AUTEUR}

\section{JEAN-CLAUDE MONNIER}

Professeur en psychopédagogie, chargé d'enseignement, IUFM de Montpellier, Maison de la Formation de Nîmes 